\title{
Divide and conquer a new parallel algorithm for the solution of a tridiagonal linear system of equations
}

\section{Report}

\section{Author(s):}

Bondeli, Stefan

Publication date:

1990

Permanent link:

https://doi.org/10.3929/ethz-a-000545862

\section{Rights / license:}

In Copyright - Non-Commercial Use Permitted

\section{Originally published in:}

ETH, Eidgenössische Technische Hochschule Zürich, Departement Informatik, Institut für Wissenschaftliches Rechnen 130 


\section{ETH}

Eidgenössische

Technische Hochschule Zürich
Departement Informatik

Institut für

Wissenschaftliches Rechnen

Stefan Bondeli

Divide and Conquer:

A New Parallel Algorithm for the Solution of a

Tridiagonal Linear System of Equations

May 1990 
Authors' address:

Wissenschaftliches Rechnen ETH-Zentrum

$\mathrm{CH}-8092$ Zurich, Switzerland

e-mail: bondeli@inf.ethz.ch

(C) 1990 Departement Informatik, ETH Zürich 


\begin{abstract}
We describe a divide and conquer algorithm which solves linear tridiagonal systems with one right-hand side, especially suited for parallel computers. The algorithm is very flexible, permits multiprocessing or a combination of vector and multiprocessor implementations, and is adaptable to a wide range of parallelism granularities. This algorithm can also be combined with recursive doubling, cyclic reduction or Wang's partition method, in order to increase the degree of parallelism and vectorizability.

The divide and conquer method will be explained. Some results of time measurements on a CRAY X-MP/28, on an Alliant $\mathrm{FX} / 8$ and on a Sequent Symmetry S81b as well as comparisons with the cyclic reduction algorithm and Gaussian elimination will be presented. Finally some numerical results are given.
\end{abstract}

\title{
1 Introduction
}

The solution of tridiagonal systems of $n$ linear equations $T \vec{x}=\vec{d}$ with one right-hand side $\vec{d}$ is a central part of many programs for scientific computation. To solve this problem on parallel computers an efficient algorithm should be available.

Divide and conquer is one of the most commonly used tools in the construction of algorithms. The basic idea underlying the technique is to solve a given problem of a certain size, by dividing the problem up into similar problems of smaller size, solving them, and then combining those solutions to form the solution of the original problem. Most commonly, the same technique is applied to the smaller problems, making the procedure recursive. Such divide and conquer algorithms are excellently suited for parallel computers.

For a scalar machine the best direct solution method is Gaussian elimination, specialized for tridiagonal systems. For vector computers, cyclic reduction which was originally proposed by Hockney and Golub [7], is more efficient, since all essential arithmetic operations can be vectorized [8, pp 286]. To solve very large systems on a vector computer, Wang's Partition Method [21] is also well suited.

On parallel computers with two CPU's the two-sided Gaussian elimination algorithm (twisted factorization [20]), discussed by Babuska [2], is very useful. The decomposition of the matrix $T$, as well as the subse- 
quent solution process, can be performed in two parallel parts. Notable among parallel algorithms is also the recursive doubling method [17]. For the special case where $T$ is symmetric and of Toeplitz form, Evans [6, pp 371] proposed a divide and conquer method (based on Cramer's Rule) to solve $T \vec{x}=\vec{d}$ on parallel computers.

In this paper, however, we describe a divide and conquer algorithm for solving general linear tridiagonal systems especially suitable for parallel computers (Multiple-Instruction-Multiple-Data systems [13, p 8]). The algorithm is very flexible, permitting multiprocessing or a combination of vector and multiprocessor implementations, and is adaptable to a wide range of the size of parallelism. (In the following sections, we refer to the new method as the "divide and conquer method" or $\mathcal{D} \mathcal{A C}(p, k)$.)

For the following considerations let us assume that the matrix $T$ is diagonally dominant (or at least positive definite), which is generally assumed for the algorithm as Gaussian elimination, cyclic reduction or Wang's partition method. This condition must be stated because of numerical stability. The algorithm which will be discussed needs, from a theoretical view, weaker assumptions.

The paper is arranged as follows: We will illustrate the algorithm with an example prior to formally presenting the algorithm. Then the divide and conquer method will be explained for any $p$, where $p$ preferably is the number of CPUs of the parallel computer and $n=k p(k, p \in \mathbb{N})$. In a third part, numerical results are reported for a FORTRAN program using the divide and conquer method. Then CPU-time measurements on a CRAY X-MP/28, on a Alliant FX/8, and on a Sequent Symmetry $\mathrm{S} 81 \mathrm{~b}$ are given and discussed. Finally we compare the CPU-times of the divide and conquer method with those of the cyclic reduction algorithm and Gaussian elimination.

\section{Divide and Conquer Method}

\subsection{Divide and Conquer Method: three parallel tasks}

Consider the tridiagonal linear system $T \vec{x}=\vec{d}$ with $n=3 k$ equations $(1<k \in \mathbb{N})$. We partition $T$ into block tridiagonal form with each diagonal block a $k \times k$ tridiagonal matrix and each subdiagonal (superdiagonal) block a $k \times k$ null matrix, except for one single nonzero element on its 
upper right (lower left) corner:

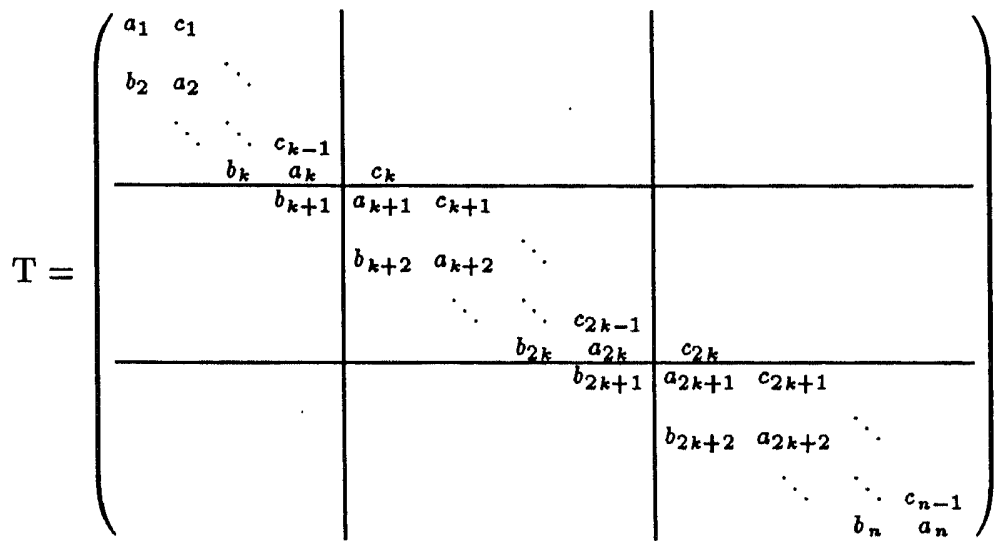

Let us suppose $b_{k+1}, b_{2 k+1}, c_{k}, c_{2 k} \neq 0$. We write the tridiagonal system in block form as

$$
\left(\begin{array}{c|c|c}
T_{1} & C_{1} & 0 \\
\hline B_{2} & T_{2} & C_{2} \\
\hline 0 & B_{3} & T_{3}
\end{array}\right)\left(\begin{array}{c}
\vec{x}_{1} \\
\hline \vec{x}_{2} \\
\hline \vec{x}_{3}
\end{array}\right)=\left(\begin{array}{c}
\frac{\vec{d}_{1}}{\vec{d}_{2}} \\
\hline \overrightarrow{d_{3}}
\end{array}\right)
$$

where $\vec{x}$ and $\vec{d}$ are partitioned to conform with $T$.

With the first and the last unit vector $\vec{e}_{1}, \vec{e}_{k} \in \mathbb{R}^{k}$, the upper- and lower-diagonal blocks of (1) are given by

$$
\begin{array}{ll}
B_{2}=b_{k+1} \vec{e}_{1} \vec{e}_{k}^{T} & B_{3}=b_{2 k+1} \vec{e}_{1} \vec{e}_{k}^{T} \\
C_{1}=c_{k} \vec{e}_{k} \vec{e}_{1}^{T} & C_{2}=c_{2 k} \vec{e}_{k} \vec{e}_{1}^{T}
\end{array}
$$

With the notation introduced above expanding (2) and rearranging, we obtain

$$
\begin{aligned}
& \vec{x}_{1}=T_{1}^{-1} \vec{d}_{1}-c_{k} T_{1}^{-1} \vec{e}_{k} \vec{e}_{1}^{T} \vec{x}_{2} \\
& \vec{x}_{2}=T_{2}^{-1} \vec{d}_{2}-b_{k+1} T_{2}^{-1} \vec{e}_{1} \vec{e}_{k}^{T} \vec{x}_{1}-c_{2 k} T_{2}^{-1} \vec{e}_{k} \vec{e}_{1}^{T} \vec{x}_{3} \\
& \vec{x}_{3}=T_{3}^{-1} \vec{d}_{3}-b_{2 k+1} T_{3}^{-1} \vec{e}_{1} \vec{e}_{k}^{T} \vec{x}_{2}
\end{aligned}
$$


By defining

$$
\begin{array}{llll}
\vec{y}_{1}=T_{1}^{-1} \vec{d}_{1} & \vec{y}_{2}=T_{2}^{-1} \vec{d}_{2} & \vec{y}_{3}=T_{3}^{-1} \vec{d}_{3} & \\
\vec{z}_{1}=T_{1}^{-1} \vec{e}_{k} & \vec{z}_{2}=T_{2}^{-1} \vec{e}_{1} & \vec{z}_{3}=T_{2}^{-1} \vec{e}_{k} & \vec{z}_{4}=T_{3}^{-1} \vec{e}_{1}
\end{array}
$$

we have the following implicit relations

$$
\begin{aligned}
& \vec{x}_{1}=\vec{y}_{1}-c_{k}\left(\vec{e}_{1}^{T} \vec{x}_{2}\right) \vec{z}_{1} \\
& \vec{x}_{2}=\vec{y}_{2}-b_{k+1}\left(\vec{e}_{k}^{T} \vec{x}_{1}\right) \vec{z}_{2}-c_{2 k}\left(\vec{e}_{1}^{T} \vec{x}_{3}\right) \vec{z}_{3} \\
& \vec{x}_{3}=\vec{y}_{3}-b_{2 k+1}\left(\vec{e}_{k}^{T} \vec{x}_{2}\right) \vec{z}_{4} .
\end{aligned}
$$

For suitable $\alpha_{i} \in \mathbb{R},(8)$, (9) and (10) can be written in the form

$$
\begin{aligned}
& \vec{x}_{1}=\vec{y}_{1}+\alpha_{1} \vec{z}_{1} \\
& \vec{x}_{2}=\vec{y}_{2}+\alpha_{2} \vec{z}_{2}+\alpha_{3} \vec{z}_{3} \\
& \vec{x}_{3}=\vec{y}_{3}+\alpha_{4} \cdot \vec{z}_{4}
\end{aligned}
$$

By substituting (11) in (4) and solving for $\vec{d}_{1}$

$$
\begin{aligned}
& T_{1} \vec{y}_{1}+\alpha_{1} T_{1} \vec{z}_{1}+c_{k}\left(\vec{e}_{1}^{T} \vec{y}_{2}\right) \vec{e}_{k}+\alpha_{2} c_{k}\left(\vec{e}_{1}^{T} \vec{z}_{2}\right) \vec{e}_{k}+\alpha_{3} c_{k}\left(\vec{e}_{1}^{T} \vec{z}_{3}\right) \vec{e}_{k}=\vec{d}_{1} \\
&\left(\alpha_{1}+c_{k}\left(\vec{e}_{1}^{T} \vec{y}_{2}\right)+\alpha_{2} c_{k}\left(\vec{e}_{1}^{T} \vec{z}_{2}\right)+\alpha_{3} c_{k}\left(\vec{e}_{1}^{T} \vec{z}_{3}\right)\right) \vec{e}_{k}=\overrightarrow{0}
\end{aligned}
$$

Since $\vec{e}_{\boldsymbol{k}} \neq \overrightarrow{0}$, the last equation implies

$$
\alpha_{1}+c_{k}\left(\vec{e}_{1}^{T} \vec{y}_{2}\right)+\alpha_{2} c_{k}\left(\vec{e}_{1}^{T} \vec{z}_{2}\right)+\alpha_{3} c_{k}\left(\vec{e}_{1}^{T} \vec{z}_{3}\right)=0 .
$$

Thus (5) is equivalent to

$$
\begin{aligned}
& b_{k+1} \vec{e}_{1} \vec{e}_{k}^{T}\left(\vec{y}_{1}+\alpha_{1} \vec{z}_{1}\right)+ T_{2}\left(\vec{y}_{2}+\alpha_{2} \vec{z}_{2}+\alpha_{3} \vec{z}_{3}\right) \\
&+c_{2 k} \vec{e}_{k} \vec{e}_{1}^{T}\left(\vec{y}_{3}+\alpha_{4} \vec{z}_{4}\right)=\vec{d}_{2} \\
&\left(\alpha_{2}+b_{k+1}\left(\vec{e}_{k}^{T} \vec{y}_{1}\right)+\alpha_{1} b_{k+1}\left(\vec{e}_{k}^{T} \vec{z}_{1}\right)\right) \vec{e}_{1} \\
&+\left(\alpha_{3}+c_{2 k}\left(\vec{e}_{1}^{T} \vec{y}_{3}\right)+\alpha_{4} c_{2 k}\left(\vec{e}_{1}^{T} \vec{z}_{4}\right)\right) \vec{e}_{k}=\overrightarrow{0}
\end{aligned}
$$

With the facts that $k>1$ and $\vec{e}_{1}, \vec{e}_{k} \neq \overrightarrow{0}$, it follows

$$
\begin{aligned}
\alpha_{2}+b_{k+1}\left(\vec{e}_{k}^{T} \vec{y}_{1}\right)+\alpha_{1} b_{k+1}\left(\vec{e}_{k}^{T} \vec{z}_{1}\right) & =0 \\
\alpha_{3}+c_{2 k}\left(\vec{e}_{1}^{T} \vec{y}_{3}\right)+\alpha_{4} c_{2 k}\left(\vec{e}_{1}^{T} \vec{z}_{z}\right) & =0 .
\end{aligned}
$$


Finally (6) and the definitions (7) imply

$$
\begin{aligned}
b_{2 k+1} \vec{e}_{1} \vec{e}_{k}^{T}\left(\vec{y}_{2}+\alpha_{2} \vec{z}_{2}+\alpha_{3} \vec{z}_{3}\right)+T_{3}\left(\vec{y}_{3}+\alpha_{4} \vec{z}_{4}\right)=\vec{d}_{3} \\
\left(\alpha_{3}+b_{2 k+1}\left(\vec{e}_{k}^{T} \vec{y}_{2}\right)+\alpha_{2} b_{2 k+1}\left(\vec{e}_{k}^{T} \vec{z}_{2}\right)+\alpha_{3} b_{2 k+1}\left(\vec{e}_{k}^{T} \vec{z}_{3}\right)\right) \vec{e}_{1}=\overrightarrow{0}
\end{aligned}
$$

and since $\vec{e}_{1} \neq \overrightarrow{0}$

$$
\alpha_{3}+b_{2 k+1}\left(\vec{e}_{k}^{T} \vec{y}_{2}\right)+\alpha_{2} b_{2 k+1}\left(\vec{e}_{k}^{T} \vec{z}_{2}\right)+\alpha_{3} b_{2 k+1}\left(\vec{e}_{k}^{T} \vec{z}_{3}\right)=0 .
$$

Equations (12), (13), (14) and (15) determine the following $4 \times 4$ tridiagonal system for the unknowns $\alpha_{i}$ :

$$
\begin{gathered}
\left(\begin{array}{cccc}
\left(\vec{e}_{k}^{T} \vec{z}_{1}\right) & 1 / b_{k+1} & 0 & 0 \\
1 / c_{k} & \left(\vec{e}_{1}^{T} \vec{z}_{2}\right) & \left(\vec{e}_{1}^{T} \vec{z}_{3}\right) & 0 \\
0 & \left(\vec{e}_{k}^{T} \vec{z}_{2}\right) & \left(\vec{e}_{k}^{T} \vec{z}_{3}\right) & 1 / b_{2 k+1} \\
0 & 0 & 1 / c_{2 k} & \left(\vec{e}_{1}^{T} \vec{z}_{4}\right)
\end{array}\right)\left(\begin{array}{c}
\alpha_{1} \\
\alpha_{2} \\
\alpha_{3} \\
\alpha_{4}
\end{array}\right)=-\left(\begin{array}{c}
\left(\vec{e}_{k}^{T} \vec{y}_{1}\right) \\
\left(\vec{e}_{1}^{T} \vec{y}_{2}\right) \\
\left(\vec{e}_{k}^{T} \vec{y}_{2}\right) \\
\left(\vec{e}_{1}^{T} \vec{y}_{3}\right)
\end{array}\right) \\
\mathcal{H} \vec{\alpha}=\vec{\beta}
\end{gathered}
$$

From the solution of (16) we then obtain the desired solution $\vec{x}$ from (10). Summarizing the divide and conquer method, which divides the given tridiagonal system $T \vec{x}=\vec{d}$ into three parallel tasks of size $k=n / 3$, can be depicted as 

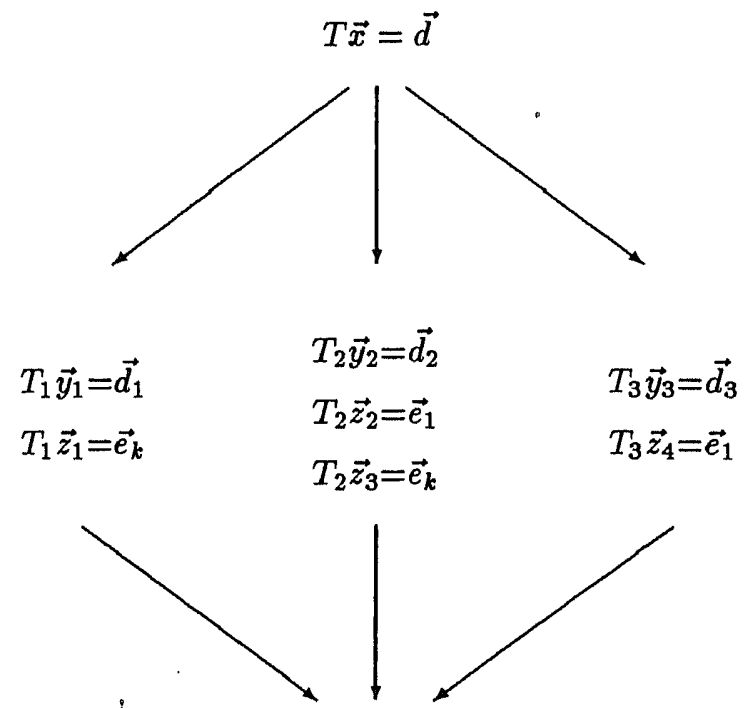

$$
\mathcal{H} \vec{\alpha}=\vec{\beta}
$$

$$
\vec{x}=\left(\begin{array}{c}
\vec{y}_{1} \\
\vec{y}_{2} \\
\vec{y}_{3}
\end{array}\right)+\left(\begin{array}{c}
\alpha_{1} \vec{z}_{1} \\
\alpha_{2} \vec{z}_{2}+\alpha_{3} \vec{z}_{3} \\
\alpha_{4} \vec{z}_{4}
\end{array}\right)
$$

and will be denoted by $\mathcal{D} \mathcal{A C}(3, k)$. 
On a parallel computer the seven systems of order $n / 3$ can be computed in parallel and independently. Actually, we have three groups of linear systems, where each group has the same coefficient matrix: two twosystem groups and one three-system group. They can be treated also parallel to each other in three parallel tasks.

\subsection{Divide and Conquer Method $\mathcal{D} \mathcal{A C}(p, k): p$ parallel tasks}

\section{Notation and Definitions}

In general, for a system of $n$ equations and for an arbitrary positive integer $p>1$, we define $k=n / p$ (assuming that $n$ is divisible by $p$ and $k>1$ ). The original tridiagonal system is then divided into square $k \times k$ blocks, analogous to (1). In block form we write

$$
\left(\begin{array}{cccc}
T_{1} & C_{1} & & \\
B_{2} & T_{2} & \ddots & \\
& \ddots & \ddots & C_{p-1} \\
& & B_{p} & T_{p}
\end{array}\right)\left(\begin{array}{c}
\vec{x}_{1} \\
\vec{x}_{2} \\
\vdots \\
\vec{x}_{p-1} \\
\vec{x}_{p}
\end{array}\right)=\left(\begin{array}{c}
\vec{d}_{1} \\
\vec{d}_{2} \\
\vdots \\
\vec{d}_{p-1} \\
\vec{d}_{p}
\end{array}\right)
$$

where $T_{i}$ are tridiagonal matrices: for $j=0,1, \ldots, p-1$

$$
T_{j+1}=\left(\begin{array}{lllll}
a_{j k+1} & c_{j k+1} & & & \\
b_{j k+2} & a_{j k+2} & c_{j k+2} & & \\
& b_{j k+3} & \ddots & \ddots & \\
& & \ddots & a_{(j+1) k-1} & c_{(j+1) k-1} \\
& & & b_{(j+1) k} & a_{(j+1) k}
\end{array}\right)
$$

and

$$
\begin{aligned}
B_{j+1} & =b_{j k+1} \vec{e}_{1} \vec{e}_{k}^{T} \\
C_{j} & =c_{j k} \vec{e}_{k} \vec{e}_{1}^{T}
\end{aligned} \quad j=1,2, \ldots, p-1
$$


assuming $b_{j k+1}, c_{j k} \neq 0(j=1,2, \ldots, p-1) . \vec{x}$ and $\vec{d}$ are partitioned to conform with the blocks of $T: \vec{x}_{j}, \vec{d}_{j} \in \mathbb{R}^{k}(j=0,1, \ldots, p-1)$

$$
\vec{x}_{j}=\left(\begin{array}{c}
x_{j k+1} \\
x_{j k+2} \\
\vdots \\
x_{(j+1) k-1} \\
x_{(j+1) k}
\end{array}\right) \quad \vec{d}_{j}=\left(\begin{array}{c}
d_{j k+1} \\
d_{j k+2} \\
\vdots \\
d_{(j+1) k-1} \\
d_{(j+1) k}
\end{array}\right)
$$

Derivation of the divide and conquer method

For $i=1,2, \ldots, p$ we define

$$
\vec{y}_{i}=T_{i}^{-1} \vec{d}_{i}
$$

and for $i=2,3, \ldots, p-1$

$$
\begin{aligned}
& \vec{z}_{m}=T_{i}^{-1} \vec{e}_{1} \\
& \vec{z}_{m+1}=T_{i}^{-1} \vec{e}_{k}
\end{aligned} \quad m=2 i-2
$$

and

$$
\vec{z}_{1}=T_{1}^{-1} \vec{e}_{k} \quad \vec{z}_{2 p-2}=T_{p}^{-1} \vec{e}_{1}
$$

$\vec{z}_{m}$ is the first and $\vec{z}_{m+1}$ is the last column of the inverse matrix of $T_{i}$.

According to (11), for suitable $\alpha_{j} \in \mathbb{R}$ the solution $\vec{x}$ can be expressed as

$$
\vec{x}_{i}= \begin{cases}\vec{y}_{1}+\alpha_{1} \vec{z}_{1} & \text { if } i=1 \\ \vec{y}_{i}+\alpha_{2 i-2} \vec{z}_{2 i-2}+\alpha_{2 i-1} \vec{z}_{2 i-1} & \text { if } 2 \leq i \leq p-1 \\ \vec{y}_{p}+\alpha_{2 p-2} \vec{z}_{2 p-2} & \text { if } i=p\end{cases}
$$

From the block system (17) and the definitions introduced above, we derive for all $j=2,3, \ldots, p-1$

$$
\begin{array}{r}
B_{j} \vec{x}_{j-1} P+T_{j} \vec{x}_{j}+C_{j} \vec{x}_{j+1}=\vec{d}_{j} \\
B_{j}\left(\vec{y}_{j-1}+\alpha_{2 j-4} \vec{z}_{2 j-4}+\alpha_{2 j-3} \vec{z}_{2 j-3}\right) \\
+T_{j}\left(\vec{y}_{j}+\alpha_{2 j-2} \vec{z}_{2 j-2}+\alpha_{2 j-1} \vec{z}_{2 j-1}\right) \\
+C_{j}\left(\vec{y}_{j+1}+\alpha_{2 j} \vec{z}_{2 j}+\alpha_{2 j+1} \vec{z}_{2 j+1}\right)=\vec{d}_{j}
\end{array}
$$


where $\alpha_{0}=\alpha_{2 p-1}=0$.

Since $T_{j} \vec{y}_{j}=\vec{d}_{j}$ and (20), (21) respectively,

$$
\begin{array}{r}
\left(b_{(j-1) k+1}\left(\vec{e}_{k}^{T} \vec{y}_{j-1}\right)+\alpha_{2 j-4} b_{(j-1) k+1}\left(\vec{e}_{k}^{T} \vec{z}_{2 j-4}\right)\right. \\
\left.+\alpha_{2 j-3} b_{(j-1) k+1}\left(\vec{e}_{k}^{T} \vec{z}_{2 j-3}\right)\right) \vec{e}_{1} \\
+\alpha_{2 j-2} \vec{e}_{1}+\alpha_{2 j-1} \vec{e}_{k} \\
+\left(c_{j k}\left(\vec{e}_{1}^{T} \vec{y}_{j+1}\right)+\alpha_{2 j} c_{j k}\left(\vec{e}_{1}^{T} \vec{z}_{2 j}\right)+\alpha_{2 j+1} c_{j k}\left(\vec{e}_{1}^{T} \vec{z}_{2 j+1}\right)\right) \vec{e}_{k}=\overrightarrow{0}
\end{array}
$$

With the facts that $k>1, \vec{e}_{1}, \vec{e}_{k} \neq \overrightarrow{0}$, and $\vec{e}_{1}$ and $\vec{e}_{k}$ are linearly independent, we know the following equations hold

$$
\begin{aligned}
& \left(\vec{e}_{k}^{T} \vec{z}_{2 j-4}\right) \alpha_{2 j-4}+\left(\vec{e}_{k}^{T} \vec{z}_{2 j-3}\right) \alpha_{2 j-3}+\frac{1}{b_{(j-1) k+1}} \alpha_{2 j-2} \\
& =-\left(\vec{e}_{k}^{T} \vec{y}_{j-1}\right) \\
& \frac{1}{c_{j k}} \alpha_{2 j-1}+\left(\vec{e}_{1}^{T} \vec{z}_{2 j}\right) \alpha_{2 j}+\left(\vec{e}_{1}^{T} \vec{z}_{2 j+1}\right) \alpha_{2 j+1} \\
& =-\left(\vec{e}_{1}^{T} \vec{y}_{j+1}\right)
\end{aligned}
$$

where $j=2,3, \ldots, p-1$.

According to (12) the first block-equation $T_{1} \vec{x}_{1}+C_{1} \vec{x}_{2}=\vec{d}_{1}$ yields

$$
\frac{1}{c_{k}} \alpha_{1}+\left(\vec{e}_{1}^{T} \vec{z}_{2}\right) \alpha_{2}+\left(\vec{e}_{1}^{T} \vec{z}_{3}\right) \alpha_{3}=-\left(\vec{e}_{1}^{T} \vec{y}_{2}\right)
$$

and the last block-equation $B_{p} \vec{x}_{p-1}+T_{p} \vec{x}_{p}=\vec{d}_{p}$ implies, like the equation (15)

$$
\begin{aligned}
\left(\vec{e}_{k}^{T} \vec{z}_{2 p-4}\right) \alpha_{2 p-4}+\left(\vec{e}_{k}^{T} \vec{z}_{2 p-3}\right) \alpha_{2 p-3}+ & \frac{1}{b_{(p-1) k+1}} \alpha_{2 p-2} \\
& =-\left(\vec{e}_{k}^{T} \vec{y}_{p-1}\right)
\end{aligned}
$$

The conditions (24), (25), (26) and (27) form a tridiagonal linear system of $2 p-2$ equations for the unknowns $\alpha_{1}, \alpha_{2}, \ldots, \alpha_{2 p-2}$.

\section{The Final Algorithm}

The divide and conquer method $\mathcal{D} \mathcal{A C}(p, k)$ for solving a tridiagonal system of $n=p k$ equations on a parallel computer with $p$ processors may be stated schematically as follows: 


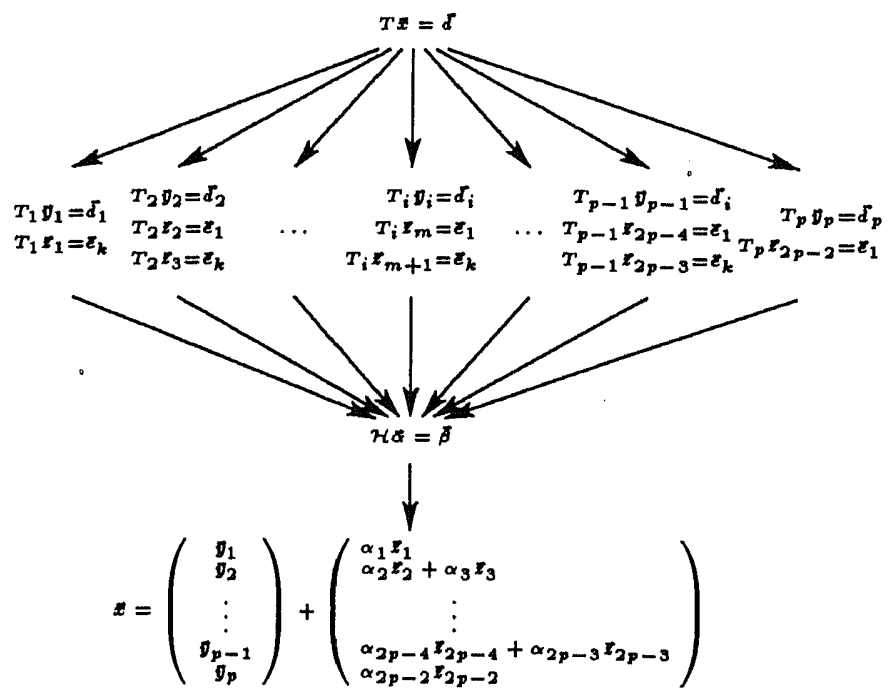

The algorithm consists of three steps:

First step: Solve in parallel the linear tridiagonal systems of size $k=n / p$ :

$$
\left.\begin{array}{ll}
T_{i} \vec{y}_{i}=\vec{d}_{i} & \text { for } i=1,2, \ldots, p \\
T_{1} \vec{z}_{1}=\vec{e}_{k} & \\
T_{i} \vec{z}_{m}=\vec{e}_{1} \\
T_{i} \vec{z}_{m+1}=\vec{e}_{k}
\end{array}\right\} \quad \text { for } i=2,3, \ldots, p-1, m=2 i-2
$$

Second step: Compute $\alpha_{i}$ by solving the tridiagonal system $\mathcal{H} \vec{\alpha}=\vec{\beta}$ 
of $2 p-2$ equations given by

$$
\left(\begin{array}{cccc}
s_{1} & t_{1} & & \\
r_{2} & s_{2} & \ddots & \\
& \ddots & \ddots & t_{2 p-3} \\
& & r_{2 p-2} & s_{2 p-2}
\end{array}\right)\left(\begin{array}{c}
\alpha_{1} \\
\alpha_{2} \\
\vdots \\
\alpha_{2 p-2}
\end{array}\right)=\left(\begin{array}{c}
\beta_{1} \\
\beta_{2} \\
\vdots \\
\beta_{2 p-2}
\end{array}\right)
$$

where the elements of $\mathcal{H}$ are defined as

$$
\begin{aligned}
s_{i} & =\left\{\begin{array}{ll}
\left(\vec{e}_{k}^{T} \vec{z}_{i}\right) & \text { if } i \text { odd } \\
\left(\vec{e}_{1}^{T} \vec{z}_{i}\right) & \text { if } i \text { even }
\end{array} \quad i=1,2, \ldots, 2 p-2\right. \\
r_{i} & =\left\{\begin{array}{ll}
\left(\vec{e}_{k}^{T} \vec{z}_{i-1}\right) & \text { if } i \text { odd } \\
1 / c_{\frac{i^{2}}{2}} & \text { if } i \text { even }
\end{array} \quad i=2,3, \ldots, 2 p-2\right. \\
t_{i} & =\left\{\begin{array}{ll}
1 / b_{\frac{i+1}{2} k+1} & \text { if } i \text { odd } \\
\left(\vec{e}_{1}^{T} \vec{z}_{i+1}\right) & \text { if } i \text { even }
\end{array} \quad i=1,2, \ldots, 2 p-3\right.
\end{aligned}
$$

and those of $\vec{\beta}$ are given by

$$
u_{i}=\left\{\begin{array}{ll}
-\left(\vec{e}_{k}^{T} \vec{y}_{\frac{i+1}{2}}\right) & \text { if } i \text { odd } \\
-\left(\vec{e}_{1}^{T} \vec{y}_{\frac{i+2}{2}}\right) & \text { if } i \text { even }
\end{array} \quad i=1,2, \ldots, 2 p-2\right.
$$

Third step: Compute the solution $\vec{x}=\left(\begin{array}{c}\vec{x}_{1} \\ \vec{x}_{2} \\ \vdots \\ \vec{x}_{p}\end{array}\right)$ by the SAXPYoperations

$$
\vec{x}_{i}= \begin{cases}\vec{y}_{1}+\alpha_{1} \vec{z}_{1} & \text { if } i=1 \\ \vec{y}_{i}+\alpha_{2 i-2} \vec{z}_{2 i-2}+\alpha_{2 i-1} \vec{z}_{2 i-1} & \text { if } 2 \leq i \leq p-1 \\ \vec{y}_{p}+\alpha_{2 p-2} \vec{z}_{2 p-2} & \text { if } i=p\end{cases}
$$

as $p$ parallel vector operations of length $k$. 


\section{Remarks}

Several observations pertinent to the divide and conquer method $\mathcal{D} \mathcal{A C}(p, k)$ can be made:

1. The divide and conquer method $\mathcal{D} \mathcal{A C}(p, k)$ works theoretically for tridiagonal matrices $T$ with the property that every single $T_{i}$ is regular. Notice that e.g. the matrices $T_{1}$ and $T_{2}$ of the regular matrix

$$
T=\left(\begin{array}{llll}
1 & 1 & 0 & 0 \\
1 & 1 & 1 & 0 \\
0 & 1 & 1 & 1 \\
0 & 0 & 1 & 1
\end{array}\right)
$$

with $\operatorname{det} T=-1$ are singular $\left(T_{1}=T_{2}=\left(\begin{array}{ll}1 & 1 \\ 1 & 1\end{array}\right)\right)$. This means that the smaller systems (28) of size $n / p=2$ can not be solved, and so the divide and conquer method breaks down.

This cannot happen however if we assume that $T$ is diagonally dominant. Then every $T_{i}$ is also diagonally dominant and thus the smaller systems can be solved.

2. To solve the system $\mathcal{H} \vec{\alpha}=\vec{\beta}$ we can again use the divide and conquer method, which makes $\mathcal{D} \mathcal{A C}(p, k)$ recursive.

3. By using Gaussian Elimination ${ }^{1}$ to solve the smaller systems (28) and $\mathcal{H} \vec{\alpha}=\vec{\beta}(29)$, the total number of scalar operations for $\mathcal{D} \mathcal{A C}(p, k)$ is $18 n+7 p-10 k-21$ (see next table). This quantity is minimized for $p=2$ and $k=n / 2$.

${ }^{1}$ Gaussian Elimination, to solve a tridiagonal linear system of $n$ equations, requires $3 n-3$ additions, $3 n-3$ multiplications and $2 n-1$ divisions [11]. 


\begin{tabular}{|l|l|l|l|}
\hline \multicolumn{4}{|c|}{ Scalar Operation Counts for $\mathcal{D A C}(p, k): n=p k$} \\
\hline $\begin{array}{l}T_{i}=L_{i} R_{i} \\
\mathrm{i}=1,2, \ldots, \mathrm{p}\end{array}$ & $n-p$ & $n-p$ & $n-p$ \\
\hline $\begin{array}{l}L_{i} \vec{u}_{i}=\vec{d}_{i} \\
R_{i} \vec{y}_{i}=\vec{u}_{i} \\
\mathrm{i}=2,3, \ldots, \mathrm{p}\end{array}$ & $2 n-2 p$ & $2 n-2 p$ & $n$ \\
\hline $\begin{array}{l}L_{i} \vec{v}_{i}=\vec{e}_{1} \\
R_{i} \vec{z}_{m}=\vec{v}_{i} \\
\mathrm{i}=2,3, \ldots, \mathrm{p}\end{array}$ & $n-p-k+1$ & $2 n-2 p-2 k+2$ & $n-k$ \\
\hline $\begin{array}{l}R_{i} \vec{z}_{m+1}=\vec{e}_{k} \\
\mathrm{i}=1,2, \ldots, \mathrm{p}-1\end{array}$ & & $n-p-k+1$ & $n-k$ \\
\hline $\begin{array}{l}1 / c_{\frac{i}{2} k} \\
1 / b_{\frac{i+1}{2} k+1}\end{array}$ & & & $2 p-2$ \\
\hline $\mathcal{H} \vec{\alpha}=\vec{\beta} \in \mathbb{R}^{2 p-2}$ & $6 p-9$ & $6 p-9$ & $4 p-5$ \\
\hline $\begin{array}{l}\vec{x}_{i} \\
\mathrm{i}=1,2, \ldots, \mathrm{p}\end{array}$ & $2 n-2 k$ & $2 n-2 k$ & \\
\hline
\end{tabular}

4. The following table gives the number of scalar operations for several known methods, that solve a single tridiagonal system of $n$ equations:

\begin{tabular}{|l|l|l|l|}
\hline & Additions & Mul. & Divisions \\
\hline Gaussian Elimination & $3 \mathrm{n}-3$ & $3 \mathrm{n}-3$ & $2 \mathrm{n}-1$ \\
\hline $\begin{array}{l}\text { Cyclic Reduction [10] } \\
n=2^{m}-1\end{array}$ & $6 \mathrm{n}-4 \mathrm{~m}-2$ & $8 \mathrm{n}-6 \mathrm{~m}-2$ & $3 \mathrm{n}-2 \mathrm{~m}$ \\
\hline $\begin{array}{l}\text { Partition Method [21] } \\
n=p k\end{array}$ & $7 \mathrm{n}-4 \mathrm{p}-3 \mathrm{k}-1$ & $9 \mathrm{n}-6 \mathrm{p}-4 \mathrm{k}-1$ & $5 \mathrm{n}-2 \mathrm{p}-\mathrm{k}-2$ \\
\hline $\mathcal{D A C}(p, k)$ & $6 \mathrm{n}+2 \mathrm{p}-3 \mathrm{k}-8$ & $8 \mathrm{n}-5 \mathrm{k}-6$ & $4 \mathrm{n}+5 \mathrm{p}-2 \mathrm{k}-7$ \\
\hline
\end{tabular}

Although $\mathcal{D} \mathcal{A C}(p, k)$ requires nearly twice the number of operations as Gaussian Elimination. $\mathcal{D} \mathcal{A C}(p, k)$ is highly parallelizable, whereas the Gauss algorithm must be executed in scalar mode. The operation count for $\mathcal{D} \mathcal{A C}(p, k)$ is nearly the same as for the classical cyclic reduction algorithm, even less than for Wang's partition method.

5. On a vector machine, an efficient implementation of $\mathcal{D} \mathcal{A C}(p, k)$ applies Gaussian Elimination to all the smaller systems of order $i$ 
simultaneously. Details, of solving simultaneously many tridiagonal systems by Gaussian elimination, and a program, written in FORTRAN, are discussed in [15].

6. The greater we choose $p$, the smaller will be the size of the smaller systems; but the larger the size of $\mathcal{H} \vec{\alpha}=\vec{\beta}$. We should choose $p$ so that on the one hand all processors of the parallel computer work as long as possible, and that on the other hand the overhead to compute $\vec{\alpha}$ is small. Furthermore we must consider that each processor can be equipped with a vector facility.

They are several possibilities to choose both the parameter $p$ and the algorithm for computing the smaller systems (28):

(a) Since $3 p-2$ linear systems of size $n / p$ must be solved, we can fix the parameter $p$ so that $3 p-2$ equals the number of CPUs of the parallel computer available. Now, three systems with every matrix $T_{i}(i=2, \ldots, p-1)$ must be solved, and so we prefer to combine the computation of $T_{i} \vec{y}_{i}=\vec{d}_{i}, T_{i} \vec{z}_{m}=\vec{e}_{1}$ and $T_{i} \vec{z}_{m+1}=\vec{e}_{k}$.

(b) The smaller systems can be solved by Gaussian Elimination where first we would compute the $L R$-decomposition of every $T_{i}$ in $p$ parallel tasks. In a second step the forwardsubstitution for the vectors $\vec{d}_{i}$ and $\vec{e}_{1}$ will be carried out in $2 p-1$ parallel tasks (Note that the forward-substitution for $\vec{e}_{k}$ is not necessary because $L \vec{e}_{k}=\vec{e}_{k}$ ). Finally, we arrive at the solutions by $3 p-2$ parallel backward-substitutions.

(c) The overhead to solve $T_{i} \vec{z}_{m}=\vec{e}_{1}$ and $T_{i} \vec{z}_{m+1}=\vec{e}_{k}$ in addition to $T_{i} \vec{y}_{i}=\vec{d}_{i}$ with cyclic reduction is small: in each reduction step we need only two elements more (one of $\vec{e}_{k}$ and one of $\left.\vec{e}_{1}\right)$. The back-substitutions, which calculate $\vec{z}_{m}$ and $\vec{z}_{m+1}$, can be performed in parallel to the one that computes $\vec{y}_{i}$.

7. Cyclic reduction is the best we can do on a vector computer with a direct solution method for a single tridiagonal system [16, $\mathrm{p} 216]$. Therefore, on a parallel computer where each CPU supports vector arithmetic (such as the Alliant FX/8), the smaller systems (28) and $\mathcal{H} \vec{\alpha}=\vec{\beta}(29)$ should be solved by using cyclic reduction. The following table lists the scalar operation counts for this case, while 
the next table lists the number of vector operations required for each step of the divide and conquer method $\mathcal{D} \mathcal{A C}(p, k)$.

\begin{tabular}{|c|c|c|c|}
\hline \multicolumn{4}{|c|}{$\begin{array}{l}\text { Scalar Operation Counts for } \mathcal{D} \mathcal{A C}(p, k) \text { by using Cyclic Reduction } \\
\qquad n=p k \quad p=2^{q-1} \quad k=2^{h}-1\end{array}$} \\
\hline & Additions & Multiplications & Divisions \\
\hline $\begin{array}{l}T_{i} \vec{y}_{i}=\vec{d}_{i} \\
i=1,2, \ldots, p\end{array}$ & $6 n-4 p h-2 p$ & $8 n-6 p h-2 p$ & $3 n-2 p h$ \\
\hline $\begin{array}{l}T_{i} \vec{z}_{m}=\vec{e}_{1} \\
\text { (unplementary to } \\
T_{i} g_{i}=\bar{d}_{i} \text { ) i=2,s, } \ldots, p\end{array}$ & $2 n-2 p-2 k+2$ & $\begin{array}{l}2 n-3 p-2 k \\
+p h-h+3\end{array}$ & $n-k$ \\
\hline $\begin{array}{l}T_{i} \vec{z}_{m+1}=\vec{e}_{k} \\
\text { (supplementary to } \\
T_{i} \nabla_{i}=\vec{d}_{i} \text { ) i=1,2, } \ldots, \mathrm{p}-1\end{array}$ & $2 n-2 p-2 k+2$ & $\begin{array}{l}2 n-3 p-2 k \\
+p h-h+3\end{array}$ & $n-k$ \\
\hline $\begin{array}{l}1 / c_{\frac{i}{2} k} \\
1 / b_{\frac{i+1}{2} k+1}\end{array}$ & & & $2 p-2$ \\
\hline $\mathcal{H} \vec{\alpha}=\vec{\beta} \in \mathbf{R}^{2 p-2}$ & $12 p-4 q-14$ & $16 p-6 q-18$ & $6 p-2 q-6$ \\
\hline $\begin{array}{l}\vec{x}_{i} \\
i=1,2, \ldots, p\end{array}$ & $2 n-2 k$ & $2 n-2 k$ & \\
\hline $\begin{array}{l}\text { Total numbers of } \\
\text { scalar operations }\end{array}$ & \multicolumn{3}{|c|}{$31 n+(22-10 h) p-14 k-12 q-30$} \\
\hline
\end{tabular}

\begin{tabular}{|c|c|c|c|}
\hline \multicolumn{4}{|c|}{$\begin{array}{l}\text { Vector Operation Counts for } \mathcal{D} \mathcal{A C}(p, k) \text { by using Cyclic Reduction } \\
\qquad n=p k \quad p=2^{q-1} \quad k=2^{h}-1\end{array}$} \\
\hline & Additions & Multiplications & Divisions \\
\hline $\begin{array}{l}T_{i} \vec{y}_{i}=\vec{d}_{i} \\
i=1,2, \ldots, p\end{array}$ & $6 p(h-1)$ & $8 p(h-1)$ & $3 p(h-1)$ \\
\hline $\begin{array}{l}T_{i} \vec{z}_{m}=\vec{e}_{1} \\
(\text { supplementary to } \\
\left.T_{i} y_{i}=d_{i}\right) \text { i }=2,3, \ldots, p\end{array}$ & $(2 p-2)(h-1)$ & $(2 p-2)(h-1)$ & $(p-1)(h-1)$ \\
\hline $\begin{array}{l}T_{i} \vec{z}_{m+1}=\vec{e}_{k} \\
\text { (supplementary to } \\
T_{i} g_{i}=\vec{d}_{i} \text { ) } i=1,2, \ldots, p-1\end{array}$ & $(2 p-2)(h-1)$ & $(2 p-2)(h-1)$ & $(p-1)(h-1)$ \\
\hline $\begin{array}{l}1 / c_{\frac{i}{2} k} \\
1 / b_{\frac{i+1}{2} k+1}\end{array}$ & & & 2 \\
\hline $\mathcal{H} \vec{\alpha}=\vec{\beta} \in \mathbf{R}^{2 p-2}$ & $6(q-1)$ & $8(q-1)$ & $3(q-1)$ \\
\hline $\begin{array}{l}\vec{x}_{i} \\
i=1,2, \ldots, p\end{array}$ & $4 p-4$ & $4 p-4$ & \\
\hline Total & $\begin{array}{l}10 p h-6 p \\
+6 q-4 h-6 \\
\end{array}$ & $\begin{array}{r}12 p h-8 p \\
+8 q-4 h-8 \\
\end{array}$ & $\begin{array}{l}5 p h-5 p \\
+3 q-2 h+1 \\
\end{array}$ \\
\hline $\begin{array}{l}\text { Total numbers of } \\
\text { vector operations }\end{array}$ & $27 p$ & $19 p+17 q-$ & 3 \\
\hline
\end{tabular}


8. In the following table, we list operation counts for some typical sizes of $n$ :

\begin{tabular}{|c|c|c|c|c|c|}
\hline \multicolumn{6}{|c|}{ Sample operation counts of $\mathcal{D A C}(p, k)$ by using } \\
\hline & & & \multirow{2}{*}{\begin{tabular}{l|l} 
& Gaussian El. \\
$k$ & Scalar count
\end{tabular}} & \multicolumn{2}{|c|}{ Cyclic Reduction } \\
\hline$n$ & $p$ & $\bar{k}$ & & Scalar count & Vector count \\
\hline 62 & 2 & 31 & 799 & 1378 & $\overline{203}$ \\
\hline 126 & 2 & 63 & 1631 & 2894 & 247 \\
\hline 124 & 4 & 31 & 1929 & 3232 & 452 \\
\hline 254 & 2 & 127 & 3295 & 5946 & 291 \\
\hline 252 & 4 & 63 & 3913 & 6712 & 550 \\
\hline 248 & 8 & 31 & 4189 & 6952 & 933 \\
\hline 510 & 2 & 255 & 6623 & 12070 & 335 \\
\hline 508 & 4 & 127 & 7881 & 13712 & 648 \\
\hline 504 & 8 & 63 & 8477 & 14360 & 1139 \\
\hline 496$]$ & 16 & 31 & 8709 & 14404 & 1878 \\
\hline 1020 & 4 & 255 & 15817 & 27752 & 746 \\
\hline 1016 & 8 & 127 & 17053 & 29256 & 1345 \\
\hline 1008 & 16 & 63 & 17605 & 29668 & 2300 \\
\hline 9923 & 32 & 31 & 17749 & 29320 & 3751 \\
\hline 2044 & 4 & 511 & 31689 & 55872 & $\overline{844}$ \\
\hline 2040 & 8 & 255 & 34205 & 59128 & 1551 \\
\hline 2032 & 16 & 127 & 35397 & 60356 & 2722 \\
\hline 2016 & 32 & 63 & 35861 & 60296 & 4605 \\
\hline 4092 & 4 & 1023 & 63433 & 112152 & $\overline{942}$ \\
\hline 4088 & 8 & 511 & 68509 & 118952 & 1757 \\
\hline 4080 & 16 & 255 & 70981 & 121892 & 3144 \\
\hline 4064 & 32 & 127 & 72085 & 122568 & 5459 \\
\hline 4032 & 64 & 63 & 72373 & 121564 & 9198 \\
\hline
\end{tabular}

Note that $\mathcal{D} \mathcal{A C}(p, k)$ using Cyclic Reduction requires approximately 1.7 times more scalar operations than when combined with Gaussian Elimination (however in the second case the vector facility on each CPU can be used). We conclude also that by fixing $n$ and increasing $p$ the vector count increases rapidly.

\section{Time Measurements}

It is assumed that the reader is familiar with the architecture of the CRAY X-MP/28, the Alliant FX/8 and the Sequent Symmetry S81b. 
For a description see [9, pp 123], [9, pp 52] or [1], [18]. The time measurements have been obtained under the following conditions:

- The $\mathcal{D} \mathcal{A C}(p, k)$ method has been coded in FORTRAN 77 and compared to both the classical cyclic reduction algorithm $\left(n=2^{k}-1\right)$ [12] and Gaussian elimination, which were also coded in FORTRAN 77. (The compiler on the CRAY X-MP/28 is CFT77 VERSION 3.1, on the Alliant FX/8 "FORTRAN, version 4.1.35" is installed and on the Sequent Symmetry S81b: FORTRAN 77 V3.0.1 (C) Copyright 1981, 1987 Silicon Valley Software Inc.)

- To force explicit parallelism and/or explicit vectorization on the CRAY X-MP/28 and on the Alliant FX/8, compiler directives were used. More detailed descriptions can be found for CRAY computers and for the Alliant in [4] and [19] respectively.

- Every smaller system-triple of the $\mathcal{D} \mathcal{A C}(p, k)$ method was combined in one single task and solved using cyclic reduction: Each processor computes

$$
T_{i} \vec{y}_{i}=\vec{d}_{i}, \quad T_{i} \vec{z}_{m}=\vec{e}_{1}, \quad T_{i} \vec{z}_{m+1}=\vec{e}_{k}
$$

(Only on the CRAY and the Alliant computers.)

- To parallelize the code on the Sequent Symmetry S81b we used functions in the Parallel Programming Library DYNIX. For more details see [14, pp 5-12].

- Since the CPUs of the Sequent Symmetry S81b do not support vector arithmetic, the smaller system-triples of $\mathcal{D} \mathcal{A C}(p, k)$ were computed using Gaussian Elimination (combined into one single task analogous to the case above).

- The system $\mathcal{H} \vec{\alpha}=\vec{\beta}$ was solved using Gaussian Elimination on all three computers.

- $\mathcal{D} \mathcal{A C}(p, k)$ always runs on $p$ processors. The number of CPU's available to the program is variable:

CRAY X-MP/28: $\quad p=1,2$

Alliant FX/8: $\quad 1 \leq p \leq 6$

Sequent Symmetry S81b: $1 \leq p \leq 25$ 
If $t(n, p)$ denotes the elapsed CPU time for the solution of a tridiagonal system of $n$ linear equations using $p$ processors always using $\mathcal{D} \mathcal{A C}(p, k)$, then we define efficiency $E(n, p)$ as a percentage

$$
E(n, p)=\frac{100 * t(n, 1)}{p * t(n, p)}
$$

If one processor takes an amount of time to do a task, then $p$ processors can do the task in the $p$ th part of the time. Only for very special situations this perfect efficiency of $100 \%$ can be achieved.

The following tables and figures show the high efficiency of $\mathcal{D} \mathcal{A C}(p, k)$ for an increasing number $p$ of processors.

\begin{tabular}{|c|c|c|c|c|}
\hline \multicolumn{5}{|c|}{ CRAY X-MP/28 } \\
\hline$n E(n, 2)$ & $\bar{n}$ & $\overline{E(n, 2)}$ & & $\overline{E(n, 2)}$ \\
\hline $6284.74 \%$ & 126 & $86.63 \%$ & 254 & $89.22 \%$ \\
\hline $510 \quad 94.55 \%$ & 1022 & $94.77 \%$ & 2046 & $94.23 \%$ \\
\hline $4094 \quad 94.99 \%$ & 8190 & $96.84 \%$ & 16382 & $98.02 \%$ \\
\hline
\end{tabular}

\begin{tabular}{|c|c|c|c|c|c|c|c|c|}
\hline \multicolumn{9}{|c|}{ Alliant FX/8 } \\
\hline & $E(n, 2)$ & $n E(n, 3)$ & & $\overline{E(n, 4)}$ & & $\overline{E(n, 5)}$ & & $E(n, 6)$ \\
\hline 126 & $96.21 \%$ & $9386.17 \%$ & 124 & $84.31 \%$ & 75 & $78.11 \%$ & 90 & $78.93 \%$ \\
\hline 254 & $97.01 \%$ & $18986.23 \%$ & 252 & $85.44 \%$ & 155 & $81.08 \%$ & 186 & 80.6 \\
\hline 510 & $97.95 \%$ & $381 \quad 86.34 \%$ & 508 & $87.15 \%$ & 315 & $83.02 \%$ & 378 & $82.14 \%$ \\
\hline 1022 & $98.22 \%$ & $76585.76 \%$ & 1020 & $87.27 \%$ & 635 & $83.95 \%$ & 762 & $82.56 \%$ \\
\hline 2046 & $98.33 \%$ & $1533 \quad 85.59 \%$ & 2044 & $86.95 \%$ & 1275 & $84.99 \%$ & 1530 & $82.82 \%$ \\
\hline 4094 & $97.66 \%$ & $306984.68 \%$ & 4092 & $79.86 \%$ & 2555 & $80.36 \%$ & 3066 & $75.81 \%$ \\
\hline 8190 & $89.17 \%$ & $6141 \quad 76.81 \%$ & 8188 & $67.24 \%$ & 5115 & $74.43 \%$ & 6138 & $64.05 \%$ \\
\hline
\end{tabular}

Figure 1: Efficiency $E(n, p)$ of $\mathcal{D} \mathcal{A C}(p, k)$ on the CRAY $\mathrm{X}-\mathrm{MP} / 28$ and Alliant $\mathrm{FX} / 8$. 

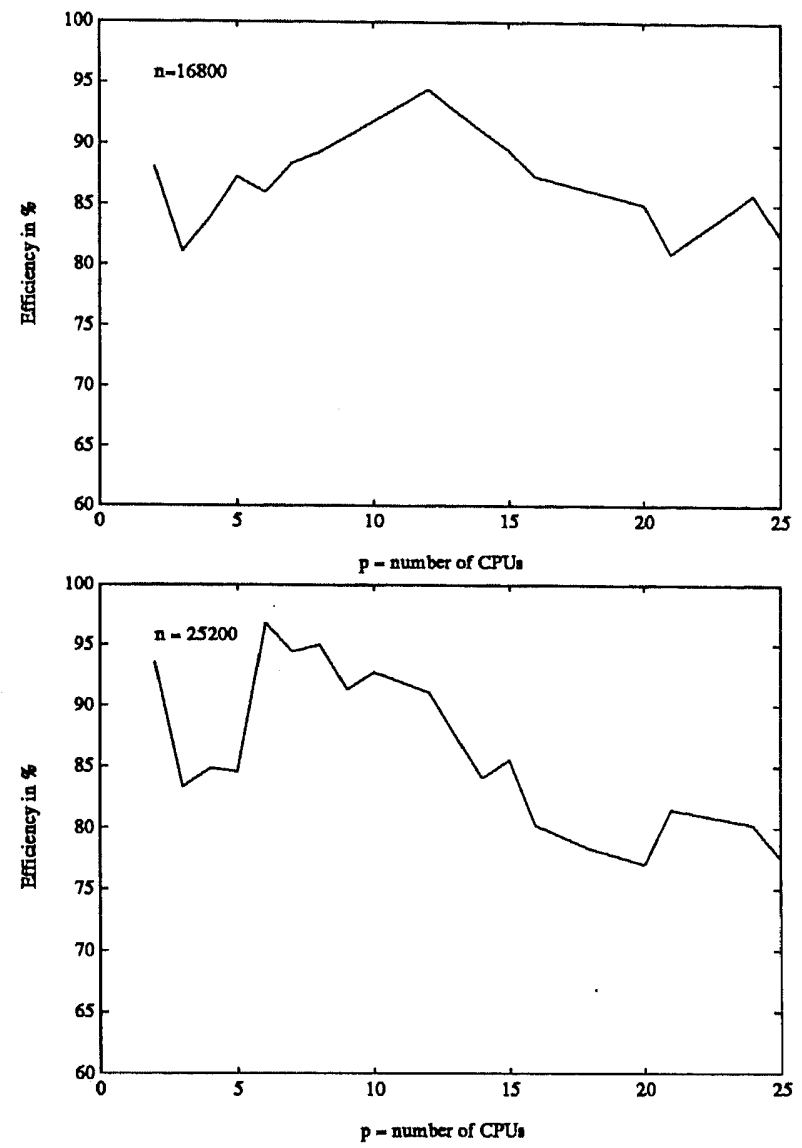

Figure 2: Efficiency $E(n, p)$ of $\mathcal{D} \mathcal{A C}(p, k)$ on the Sequent Symmetry S81b for $n=16800$ and $n=25200$.

The efficiency on the CRAY X-MP/28 grows with an increasing size $n$ until reaching a maximum value of $96.84 \%$. On the Alliant $\mathrm{FX} / 8$ the maximum value of $E(n, p)$ decreases slowly with an increasing number of CPUs $p$. This is attributed to the fact that the size of the system $\mathcal{H} \vec{\alpha}=\vec{\beta}$ increases (It was computed on one single CPU). For this same reason the efficiency on the Sequent decreases rapidly for $p>10$. The maximum value on this machine is around $95 \%$. 
The speedup reported in the following figures is given by

$$
\text { Speedup }=\frac{t_{\text {algorithm }}(n, 1)}{t_{\mathcal{D A C}(p, k)}(n, p)}
$$

where $t_{\text {algorithm }}(n, 1)$ denotes the measured time for the cyclic reduction or Gaussian elimination on one processor.
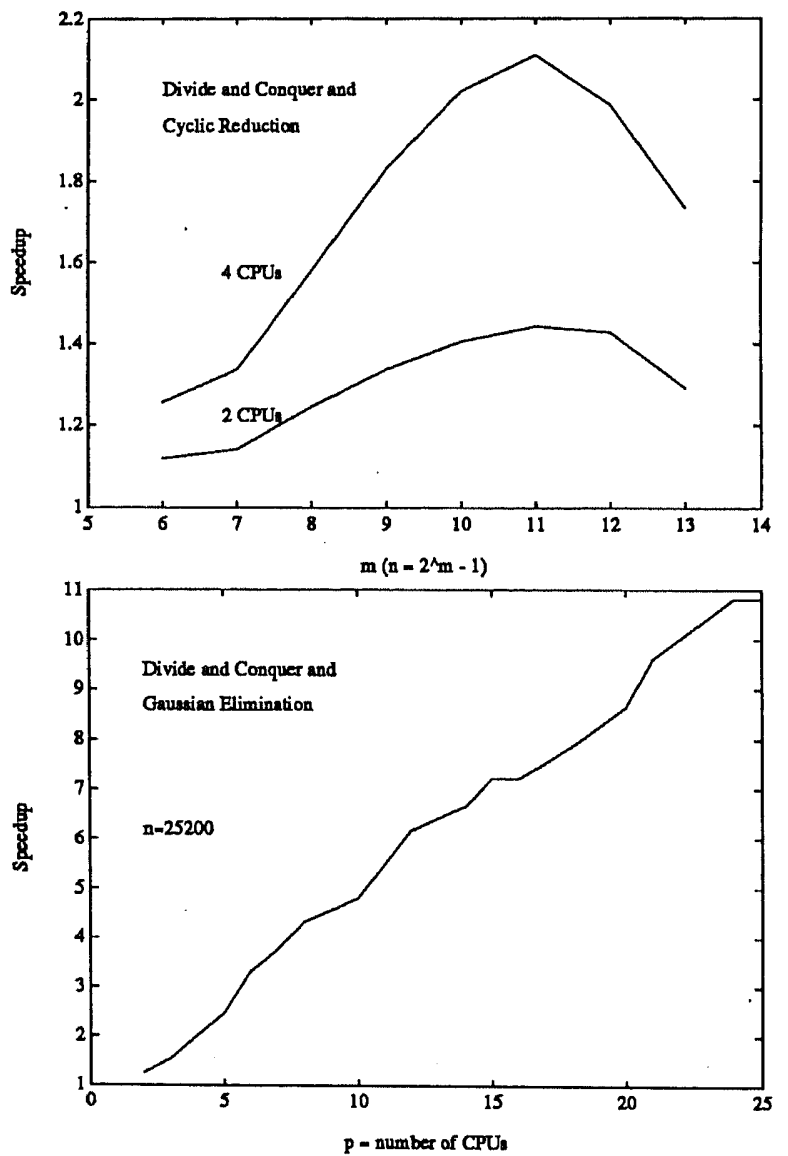

Figure 3: Speedup of $\mathcal{D} \mathcal{A C}(p, k)$ on the Alliant FX/8 (above) and on the Sequent Symmetry S81b (below). 
The maximum speedup of $\mathcal{D} \mathcal{A C}(p, k)$ and cyclic reduction on the Alliant FX/8 is approximately 1.4 using 2 CPUs and 2.1 using 4 CPUs - this occurs at $n=2^{11}-1=2047$. So we gain about a factor of 2 on the method that was rated best until now.

The speedup of $\mathcal{D} \mathcal{A C}(p, k)$ and Gaussian Elimination on the Sequent grows with an increasing number of CPUs until reaching its maximum value at about 10.8 (on $25 \mathrm{CPUs}$ ).

\section{$5 \quad$ Numerical examples}

Finally, we present a few examples that show the accuracy of the divide and conquer method $\mathcal{D} \mathcal{A C}(p, k)$ by using Gaussian Elimination to solve the smaller system-triples.

The algorithm has been coded in FORTRAN and the observed error norms are given for execution on a CRAY X-MP/28 on only one processor (which is equivalent to the accuracy on more than one processor). All computations have been carried out in floating-point arithmetic with a relative accuracy of 48 bits (approximately 14 decimal digits of precision $[5$, pp 3-6]).

We set $n=4000, a_{i}=a$, and $b_{i}=c_{i}=-1$. The right-hand side $\vec{d}$ is chosen such that the exact solution is $x_{i}=i / 20$. The computed solution is denoted by $\overrightarrow{\hat{x}}$. The entries in the following table are the relative maximum errors $\varepsilon_{p}$ given by

$$
\varepsilon_{p}=\max _{1 \leq i \leq 4000} \frac{\left|\hat{x}_{i}-x_{i}\right|}{\left|x_{i}\right|}
$$

where $p$ equals the number of system-triples of $\mathcal{D} \mathcal{A C}(p, k)$.

\begin{tabular}{|r|ccc|}
\hline$p$ & $a=4.0$ & $a=2.0$ & $a=1.9$ \\
\hline 25 & $0.201587 E-13$ & $0.515926 E-08$ & $0.145070 E-08$ \\
16 & $0.184357 E-13$ & $0.528623 E-08$ & $0.145288 E-09$ \\
10 & $0.194060 E-13$ & $0.516068 E-08$ & $0.243210 E-08$ \\
8 & $0.182081 E-13$ & $0.513520 E-08$ & $0.454429 E-08$ \\
4 & $0.182081 E-13$ & $0.501056 E-08$ & $0.439423 E-08$ \\
2 & $0.141446 E-13$ & $0.475870 E-08$ & $0.286127 E-08$ \\
\hline \hline Gauss: & $0.142053 E-13$ & $0.535147 E-08$ & $0.117544 E-07$ \\
\hline Cycl. Red.: & $0.464028 E-13$ & $0.751175 E-08$ & $0.413403 E-09$ \\
\hline tor n=409s
\end{tabular}


The last two rows show the errors, occurring in the solution of the tridiagonal linear system by using both Gaussian Elimination and Cyclic Reduction.

We observe that for the model problem $a=4.0$, the relative error norms are much smaller than for the ill conditioned cases $a=2.0$ and $a=1.9$. Other values for $|a|>2$ lead to results similar to those in the first column of the table above.

Strikingly, for $T$ diagonally dominant, the relative maximum error by the Cyclic Reduction algorithm is more than twice that of $\mathcal{D} \mathcal{A C}(p, k)$.

In the first two examples $\mathcal{D} \mathcal{A C}(p, k)$ and Gaussian Elimination have nearly the same error. For the case $a=1.9$, however, $\mathcal{D} \mathcal{A C}(p, k)$ is a little better.

The accuracy of the solution $\overrightarrow{\hat{x}}$ is mainly determined by the computed solutions $\vec{y}_{i}$ and $\vec{z}_{i}$ of the smaller systems, and for values $|a|<2$, pivoting may be necessary!

Acknowledgements The author is indebted to G. Golub (Stanford University) and W. Gander (ETH Zürich) for many discussions, comments, and suggestions regarding this paper. Help for time measurelents was provided by W. Petersen and R. Rühl (ETH Zürich). Thanks sr proofreading the paper goes to L. Pfau (ETH Zürich). 


\section{Literatur}

[1] Annaratone M., Rühl R.: Performance measurements on a commercial multiprocessor running parallel code, Technical Report No. 88/18, Institut für Integrierte Systeme, ETH Zürich 1988

[2] Babuska I.: Numerical stability in problems of linear algebra, SIAM J. Numer. Anal. 9 (1)(1972) 53-77.

[3] Bondeli S.: A new Parallel Algorithm for the Solution of a Tridiagonal Linear System of Equations, Proceedings of the Joint Conference CONPAR 90/VAPP IV, to appear

[4] CRAY COMPUTER SYSTEMS, CFT77 Reference manual SR0018, CRAY Research, Inc. 1986.

[5] CRAY X-MP and CRAY-1 COMPUTER SYSTEMS, Fortran (CFT) Reference manual SR-0009, CRAY Research, Inc. 1986.

[6] Evans D.J.: Parallel Processing, Cambridge University Press, Cambridge 1982 .

[7] Hockney R.: A Fast Direct Solution of Poisson's Equation using Fourier Analysis, J. ACM 12 (1965) 95-113.

[8] Hockney R., Jesshope C.: Parallel Computers, Adam Hilger Ltd., Bristol, 1981.

[9] Hockney R., Jesshope C.: Parallel Computers 2, Adam Hilger Ltd., Bristol, 1988.

[10] Johnsson S.L.: Solving tridiagonal Systems on ensemble Architectures, SIAM J. SCI. STAT. Comput., Vol. 8, No 3, (May 1987) 354-389 .

[11] Lakshmivarahan S., Dhall S.K.: A new class of parallel algorithms for solving linear tridiagonal systems, 1986 315-324.

[12] Lambiotte J., Voigt R.: The Solution of Tridiagonal Linear Systems on the CDC STAR-100 Computer, ACM Trans. Math. Soft. 1, 308-329.

[13] Ortega J., Introduction to Parallel and Vector Solution of Linear Systems, Plenum Press, New York 1988 
[14] Osterhaug Anita, Guide to Parallel Programming on Sequent Computer Systems, Sequent Computer Systems, Inc. 1987.

[15] Petersen W.P.: Vector Fortran for Numerical Problems on CRAY1, Communications of the ACM 26 (11)(1983) 1008-1021

[16] Schoenauer W.: Scientific Computing on Vector Computers, NorthHolland 1987.

[17] Stone H.S., Parallel tridiagonal equation solvers ACM Trans. Math. Softw. 1 (4)(1975) 289-307.

[18] Symmetry Technical Summary, Sequent Software Manual 100344447, Sequent Computer Systems, Inc. 1987 .

[19] Using the Alliant FX/8, Mathematics and Computer Science Division Technical Memorandum No. 69, Rev. 2, Vol. 1, ARGONNE NATIONAL LABORATORY, November 1987.

[20] van der Vorst H.A.: Large tridiagonal and block tridiagonal linear systems on vector and parallel computers, Parallel Computing 5 (1987) 45-54.

'21] Wang H.H.: A Parallel Method for Tridiagonal Equations, ACM Trans. math. Software 7 (2)(1981) 170-183. 
Gelbe Berichte des Departements Informatik

117 N. Wirth

118 H.-J. Schek

H.-B Paul

M.H. Scholl

G. Weikum

119 J. Gutknecht

120 D. Mey

121 H.P. Frei

P. Schäuble

M.F. Wyle

122 P. Läuchli

123 B. Wüthrich

24 C. Pfister

125 R. Crelier

126 A. Szyperski

127 H. Mössenböck

128 B. Sanders

129 L. Pfau
Modula-2 and Object-Oriented Programming. Drawing Lines,Circles, and Ellipses in a Raster. Flintstone.

The DASDBS Project: Objectives, Experiences, and Future Prospects

The Oberon Guide

A Predicate Calculus with Control of Derivations

The Assessment of Information Retrieval Algorithms

An Elementary Theory for Planar Graphs

Detecting Inconsistencies in Deductive Databases

The Graphics Editor Condor

The Layout System Pedro

OP2: A Portable Oberon Compiler

Network Communication in the Oberon Environment

Coco/R: A Generator for Fast Compiler FrontEnds

Eliminating the Substitution Axiom from UNITY Logic

GFT: A Tool for Data Management in the UNIX Environment 